\title{
REPRÄSENTATIONSRÄUME: KLEINE UTOPIEN UND WEIBLICHE FLUCHTEN. GROTESKEN IM HIPHOP-CLIP
}

\author{
Birgit Richard
}

Dieser Beitrag konzentriert sich in der Analyse der Bilder auf die Konstruktion der Identität des schwarzen Subjekts und seine sozialen Bedingungen im HipHop. Dabei ist ein Fokus die Darstellung von Geschlecht und die Suche nach emanzipatorischen Bildern von Weiblichkeit im zeitgenössischen Clip. HipHop-Videos bieten in ihrer mittlerweile sehr ausdifferenzierten Vielfalt auch unter scheinbar sexistischen Oberflächen Spielraum für weibliche und geschlechtlich nicht eindeutige Konstruktionen. Die gewählten Beispiele zeigen, neben dem kritischen Umgang mit den Klischees des Gangsta-Rap, kleine weibliche Fluchten. In Kontrast hierzu tritt eine Parodie, konstruiert aus der Perspektive weißer Rezipienten.

Die Analyse der drei im weitesten Sinne als HipHop-Videos zu bezeichnenden Clips findet unter bestimmten Voraussetzungen statt: die Unmöglichkeit den eigenen festgelegten Standpunkt als weiße( $r$ ) Interpretierende(r) zu verlassen und die sich daraus ergebende Problematik der gebrochenen Rezeption. Diese wird überdeutlich am Beispiel eines Videos von Aphex Twin, das in einen spannungsreichen Kontrast zu den Clips von Busta Rhymes und Brandy tritt. Jeder Analyse von Videos aus dem HipHop- und R'n'B-Genre muss zugrunde gelegt werden, dass bestimmte Posen und Ausdrücke auf rhetorischen Strategien basieren wie dem »signifying monkey«, Oberbegriff für das Zusammenspiel aller rhetorischen Figuren: Wörterverdrehung, Wiederholung und Umkehrung. Bei vielen Ausdrücken muss das ganze Diskursuniversum mitbedacht werden, um den Sinn zu begreifen. HipHop ist nicht einfach ein Revival afro-amerikanischer Traditionen (Musik, oral traditions), sondern repräsentiert eine zweite Stufe medienvermittelter Erzählkunst (Rose, zit. n. Mc Laren 1995: 9). Die Themen des HipHop sind "appropriation « von Zitaten aus der Bildwelt der Medien (Fernsehshows, berühmte Sportler, Videospiele und Markenartikel; Shustermann 1994: 169f.).

Auf der bildlichen Ebene lassen sich in den Musikvideoclips vier inhaltliche Grundmuster und ihnen zugeordnete Stereotypen feststellen: 
1. Gangsterism: Das Rumhängen mit den Homies, den Homeboys, das sind die Kumpel, in der Hood, der Nachbarschaft, neighbourhood). Gezeigt werden Langeweile, Überfälle, Gangbanging = Schießereien, Schlägereien, Drogendeals, Glücksspiel und Posing mit der eigenen Gang. Die Szenarien sind aggressiv: geballte Fäuste und verhüllte Gesichter der Gangsta, Waffen, Kampfhunde. Feuer und Zerstörung erhellen die meist dunklen, zerfallenen Räume, in denen sich die Gangsta präsentieren. Ihre Repräsentation unterteilt sich nochmals in Typen von Kriegern, Kids und Machos.

2. Luxus und Konsumwelten: Der erfolgreiche Gangsta präsentiert als Attribute für seinen Status die Villa, den Pool, die Frauen, die Autos: europäische Marken (Mercedes, BMW, Ferrari) sowie amerikanische Jumpcars und Lowridings, Handys, Badewannen, Whirlpools, luxuriöse Innenräume, Glücksspiel, Zigarren, Goldschmuck, übersprudelnde Champagnerflaschen als Symbole für unzählige ungezügelte Orgasmen (siehe Videos von LL Cool J, Dr. Dre, Nelly, Big PUN) und unzählige bereitwillige, knapp bekleidete Frauen. Partyszenen, die oft auch im Block, im öffentlichen Raum stattfinden, sind zu unterteilen in Parties mit den Homies auf der Straße der eigenen Neighbourhood, am Pool, oder Konzerte, wo die (eigenen) Frauen unter Umständen ganz fehlen oder Parties mit der Großfamilie, wie Barbequeues in öffentlichen Parks (z.B. Jazzy Jeff and The Fresh Prince: »Summertime $«)$.

3. Die Schattenseiten des Gangstertums: Tod, Beerdigung, Gefängnis, Polizei, verwaiste Kinder, verwitwete Frauen (Ice Cube, Nonchalant: »Five O'Clock In The Morning «) werden als Folge der Unterdrückung und Chancenlosigkeit gegenüber den weißen Autoritäten dargestellt. Seltener sind die Clips, die vor dem Wahnsinn der gegenseitigen Auslöschung schwarzer männlicher Jugendlicher warnen. Die mahnenden Stimmen kommen meist von den weiblichen Rappern, die ihre tragische Rolle der Frau als Trauernde und als Zurückgebliebene nicht länger hinnehmen wollen.

4. Der springende, wahnsinnig gestikulierende »verrückte Nigga«, der sich in Phantasiewelten anderer Art als den Luxuswelten bewegt, in imaginären, silbern-futuristischen, märchenhaften Szenerien oder in Comicwelten. Eine der ersten Figuren dieser Art ist Flavor Flav von Public Enemy. Ein Mitglied des Wu-Tang Clans, RZA, präsentiert sich als Comicfigur Bobby Digital. Die Szenen, in imaginären Räumen, als auch die Verkleidung der Verrückten sind oft durch Grundfarben gestaltet. 
In den Kategorien eins bis drei werden Ausschnitte aus dem afro-amerikanischen Leben, der »afro-diasporic culture« (Tricia Rose) am Rand eines postindustriellen, urbanen Amerika gebrochen widergespiegelt. Die HipHopVideos versuchen die Diskriminierung der schwarzen BürgerInnen in der USamerikanischen Gesellschaft sichtbar zu machen bzw. imaginär aufzuheben.

Die Szenen, in denen Extremsituationen wie Gangbanging oder Ausnahmen vom Alltag, wie Parties, gezeigt werden, machen auf weiße Betrachterlnnen den Eindruck wenig alltäglicher Begebenheiten. Das Prinzip des »living on the edge «, eines Lebens zwischen Gefahr und Vergnügen, eine durchaus alltägliche Situation für schwarze männliche Unterschichtsjugendliche, wird hier visualisiert. Für diese Jugendlichen, die in den USA sonst übersehen oder zum Schweigen gebracht werden, konstruiert Rap den männlichen schwarzen Körper als Ort des Vergnügens und der Macht. Der schwarze junge Mann stellt sich als gefährlich und begehrenswert dar (Hooks 1992: 35f.). Das ständige Gefühl der Inferiorität gegenüber den Weißen führt dazu, dass die schwarzen Männer betont männliche Charakteristika herauskehren: demonstrative Sexualität, körperliche Potenz und kriegerisches Verhalten (Wallace 1993: 57). Negative Konnotationen wie Faulheit und Gewalttätigkeit werden zu positiven Attributen von Stärke und dienen dem Widerstand gegen weiße Beherrschung (McLaren 1995: 17). Die Videos zeigen exzessiven Konsum von Luxusartikeln. Teure Autos, Kleidung und High-Tech-Geräte sind Statussymbole desjenigen, der es geschafft hat. In der Mode beginnt HipHop nicht von ungefähr in den 1980er Jahren mit dem ungenierten Zitieren der elitären Zeichen der Haute Couture, die einfache prägnante Logos besitzt (umgehängte Mercedes-Sterne, das Gucci- oder Chanel-Label auf T-Shirts; zur Mode im Allgemeinen siehe Richard 1998). Die Zeichen des unerreichbaren Wohlstandes und der Macht werden durch »blow up«-Verfahren übertrieben. Die schwarze HipHop-Kultur nimmt sich etwas, was ihr in den Augen einer weißen Mittelklasse nicht zusteht. Das Zitieren der Traumbilder und ihre Transformation über HyperkonsumStrategien zeugen von der Unerfüllbarkeit dieses Wunsches auf der Grundlage der sozialen Situation in den black communities. Tricia Rose (1994a: 80) interpretiert die exzessive Betonung von Konsumgütern im HipHop-Stil als Mittel, um auf Schichtunterschiede und Hierarchien anzuspielen und kulturelles Terrain zu erobern. Die Repräsentation von Konsummacht wird zum Mittel kulturellen Ausdrucks, die auch durch Fake oder illegale Tätigkeiten bewerkstelligt werden kann.

Die Attribute des erfolgreichen Gangsters zeigen neben der Verlockung aber auch die Auswirkungen von Gangbanging und Kriminalität, den fatalen Kreislauf von Gefängnis und Tod (Shustermann 1994: 171). Der erfolgreiche 
Rapper als einer, der diesem Ghettokreislauf entronnen ist und sich trotzdem mit den gleichen Attributen schmücken kann (Ice T, Ice Cube), bietet sich als Ersatz-Identifikationsfigur an. Sein Selbstlob stellt neben seiner sexuellen Attraktivität den kommerziellen Erfolg und seine persönlichen Vorzüge, seine Fähigkeit zu reimen, in den Mittelpunkt (Shustermann 1994: 159).

Handlungsort ist der urbane Raum der Metropolen: sowohl die heruntergekommenen, verfallenen Straßen in der New Yorker Bronx und in Brooklyn oder in Detroit, in denen die Videos der East Coast Gangsta-Rapper spielen (Mitte der 1990er z.B. Onyx, Black Sheep, House of Pain, aktuell Eminem), als auch die auf den ersten Blick sehr adretten sonnigen Hoods mit den Einfamilienhäusern in Stadtteilen wie Compton in Los Angeles, die aber nicht minder gefährlich sind, als typische Bilder von der West Coast (Ice T, Snoop Doggy Dog). Der Ort, wo sich dieses alltägliche Leben gerade abspielt, soll präzise festgehalten werden, deshalb kommen oft Straßenschilder ins Bild. Mehrere Stufen von lokalem Bezug werden gezeigt, abstrakte Regionalität mit der Zuordnung zu East oder West Coast und gleichzeitig die konkrete Regionalität (»Straight Outta Compton«, ein Titel von NWA, Niggas with Attitude). Schrift und Schriftzüge in Form von Graffiti zeigen gleichzeitig die Globalität des Stils. Die Videos beschränken sich auf Bilder aus den black communities. Weiße tauchen als neutrale Figuren nur in den Party Crowds auf. Meist sind sie RepräsentantInnen weißer Macht, z.B. Polizisten, die entweder besonders brutal oder dumm dargestellt werden.

In den Videobeiträgen dokumentiert sich auch eine massive Diskriminierung von Frauen. Die Frau wird oft als Besitzstand neben Autos und anderen Luxusobjekten präsentiert. Wenn die so funktionalisierten Frauen mit Stolz ihren Körper präsentieren, dann liegt das laut Bell Hooks (1993: 43f.) daran, dass sie so erzogen werden, dass ihnen (geistige) Alternativen zur Rolle des dienenden Körpers vorenthalten werden. Hooks stellt bei ihrer Suche nach Gründen für die geringe Zahl schwarzer weiblicher Intellektueller fest, dass nur zwei Frauenstereotypen in der afro-amerikanischen Kultur existieren: die Hure/Schlampe (bitch) oder die nährende, sorgende Mammy (vgl. auch Decker 1994: 110). Die schwarze Frau erhält aus den HipHop-Videos die Aufforderung sich in die Rolle einzufügen, die ihr vor allem die Anhänger der konservativen Nation of Islam unter Louis Farrakhan und die Ursprungsmythen der fruchtbaren Urmutter Isis zugedacht haben: Hausfrau und Mutter (Decker 1994: 107ff.). Schwarze Kulturkritiker kritisieren die sexistische Ausrichtung des HipHop. Greg Tate (1993: 226f.) hält das »Black Male Posturing « (das Posieren) für die kulturell notwendige Basis von Basketball, Jazz und HipHop und hält diese für Stilattitüden. Er kritisiert die phallozentris- 
tische Ausrichtung des schwarzen Nationalismus, da sie nur von den wahren Problemen, wie der alltäglichen Unterdrückung, ablenke (siehe auch Julien 1993: 218). Der Ursprung des Sexismus des schwarzen Mannes liegt für alle diese Kulturkritiker im weißen Rassismus. Der Selbsthass der Schwarzen führe dazu, dass schwarze Männer schwarze Frauen hassen (Wallace 1993: 57, vgl. auch Hooks 1993: 43). Es finde eine Verlängerung weißer Stereotypen statt. Kritik an dieser Inferioritäts- und Selbsthass-These übt Stanley Crouch (1993: 152), indem er behauptet, der Selbsthass der schwarzen Amerikaner sei eine ethnische Variante der Unzufriedenheit mit den Schönheitsstandards der jeweiligen Zeit. Bei den Weißen würde ein ähnlicher Wunsch nach Veränderung des Körpers der Schönheitschirurgie die Kunden in die Arme treiben. HipHop weist Frauen mit Ausnahme von HipHop-Queens wie Latifah eine marginale, nicht stilbildende Rolle zu. Die Produzentin Missy Elliott durchbricht mit real und medial beeindruckender Körpermasse das Prinzip massiver männlich-stofflicher Präsenz. Sonst sind die Frauen in den Videos als stigmatisierte »bitches « fast textillos, während die Männer unter Kamera-Untersicht bildschirmfüllende Masse demonstrieren, mit voluminöser Kleidung oder großem Körperumfang (Big Punisher, Notorious B.I.G.). Reales Pendant auf der Straße ist die Erweiterung der männlichen Körpersilhouette zur Drohgebärde (zum Prinzip des Dread vgl. Hebdige 1981). Das beliebte Oversize-Prinzip und die grelle Grundfarbigkeit der Kleidung dient der Abschreckung möglicher Gegner auf der Straße. Konkreter gesellschaftlicher Straßen-Raum wird auch besetzt durch die Graffitis als Zeichen von Präsenz und die Kultivierung einer bestimmten Art schleppend, seitlich raumgreifend zu gehen. Im Video wird dies noch verstärkt durch die umspannende Gestik der Rapper, wiederum unterstützt durch Fischauge und Froschperspektive, die den Körper raumumspannend werden lassen. Der Gang wird im Video zu einer Vor- und Rückwärtsbewegung frontal zur Kamera, die im extremen Close-up kulminiert. Dieses entsteht jedoch selten durch die Bewegung der Kamera oder durch Zoomen, sondern durch die Bewegung des Bildgegenstandes.

Die Repräsentations-Bilder in den Clips und auf der Straße erwecken den Eindruck eines direkten >dokumentarischen< Transfers von der materiellen in die virtuelle Realität. Es entsteht eine permanente Wechselwirkung zwischen diesen beiden Oberflächen. Bestimmte Stilmerkmale lassen sich wiederum schlecht übertragen, wie die verschärfte Faltenbildung im Kleidungsstil bei Baggy-Pants und dem einen hochgeschobenen Hosenbein, da die mediale Ästhetik des HipHop zwar voluminöse, pseudodreidimensionale, aber nur glatte Oberflächen darstellen kann. 
Eine werkimmanente Analyse von HipHop-Videos gestaltet sich sehr ambivalent und schwer, da in der Mehrzahl der Clips immer noch gewalttätige und sexistische Bilder-Klischees vorherrschen. Es stellt sich die Frage, ob die oben genannten sexistischen und manchmal auch rassistischen Untertöne der Rapper, die sich offen gegen Frauen, Schwule und Juden aussprechen, nur aus Kommerzgründen wiederholt werden oder ob sie auch auf bestimmte Strömungen in der Szene selbst verweisen. Das Black Business, Plattenfirmen wie Def Jam, schwarze Produzenten wie Dr. Dre oder Rapper wie LL Cool J vermarkten die Gangsta-Attitude offensiv, da sie sich gut verkauft (Rose 1994b: 124). Diese Feststellung lässt sich auf der Grundlage einer werkimmanenten Betrachtung trotz des Bewusstseins, dass aus der Perspektive von weißen Rezipienten interpretiert wurde, nicht verdrängen.

\section{»How to represent«. Stil in der Clip- ̈̈sthetik}

HipHop ist keine klassische Arbeiterjugendkultur, sondern die kulturelle $\ddot{A} u-$ ßerung von gesellschaftlich marginalisierten Jugendlichen im urbanen Raum der Metropolen. Die Binnendifferenzierung innerhalb des HipHop drückt sich über die visuellen Mittel aus. Je nachdem, ob es sich um G(angsta)-Funk oder um P(arty)-Funk handelt, variieren Setting und Gestaltungsmittel. Die Clips sind bis zur Mitte der 1990er Jahre selten durch elektronische Verfremdungen, Ausschnitthaftigkeit oder harte, schnelle Schnitte gestaltet. Sampling- und Scratch-Techniken werden nicht auf die visuelle Ebene übertragen. Experimentelle Videos begleiten meist einen verschachtelten Reimstil (Leaders of the New School: Busta Rhymes, Pharcyde, Ol' Dirty Bastard). Rückblenden werden ausschließlich für dokumentarische Szenen aus der Vergangenheit benutzt, wie Ausschnitte von Filmdokumenten von Martin Luther King, Malcolm X, oder die Darstellung schwarzer (Unterdrückungs)Geschichte, wie z.B. Filme von Sklaventransporten oder prügelnden Polizisten. Die Clips folgen narrativen Strukturen und laufen in Echtzeit. Sie wirken dokumentarisch, wie eine Liveübertragung aus den Neighbourhoods. Die Bilder sind ein Pendant dessen, was Ice $T$ »reality rap « nennt (McLaren 1995: 38). Die Beschränkung auf Schwarz-Weiß-Bilder verleiht den Videos Authentizität und verdeutlicht die Härte der Straße. Verfremdungen und Überblendungen wie schwarze Balken oder unscharfe Stellen im Bild tauchen vor allem in Gangsta-Rap-Videos auf, um Gewalt oder Waffen für die Zensur zu verschleiern, obwohl die Szenen kein Hehl daraus machen, worum es geht. Bei den Gangsta-Videos befindet sich die Kamera gerne in der 
Froschperspektive, um den Respekt vor dem Gangster auch formal auszudrücken.

In der Gegenwart haben sich HipHop-Videos weiter ausdifferenziert und digitale Mittel werden verstärkt eingesetzt. Beispielhaft sind hierfür die Videos von Missy Elliott und Busta Rhymes, die eher die ausgeflippten Charaktere repräsentieren. Ein wesentliches Stilmittel ist bei innen die Verzerrung durch das Fischauge, eine $180 \mathrm{Grad}$ Weitwinkel-Ästhetik.

Einzelbilder und Erzählung müssen immer wieder an den Kontext der sonstigen Darstellungsschemata im HipHop angebunden werden, das heißt, die folgende werkimmanente Analyse wird immer wieder in den oben erwähnten sozialen Hintergrund gestellt.

\section{Busta Rhymes: »Get Out« (2000)}

Busta Rhymes' Video »Get out « stellt auf den ersten Blick eine Mischung von typischen Videoplots für HipHop dar. Es weicht aber vor allem in seiner kritischen Aussage gegen die Bildwelten des Gangsta-Rap von gängigen Schemata ab und es unterscheidet sich auch von Busta Rhymes' bisherigen Videos, die ausschließlich inn als sverrückten Nigga in den Vordergrund stellen. Die am Anfang des Videos vorherrschende >Angeber<- und LuxusÄsthetik wird schon in der ersten Szene durch die Anwesenheit eines Kindes gebrochen, das respektlos über die teure Einrichtung der weiß getünchten Schloss-Imitation tobt. Im Hintergrund der Szene ist eine goldene Schallplatte zu sehen, die den persönlichen Bezug zum Rapper herstellt und Authentizität konstruiert. Der Sänger Busta Rhymes tritt nach einer Neckerei mit dem Kind aus der Tür des weißen Schlosses und steigt die Treppe hinab. Die Kamera verharrt in Untersicht am Treppenabsatz. Er ist weiß gekleidet, trägt eine Sonnenbrille, seine weiße Jeansjacke ist offen und er zeigt nackte Haut. Er besteigt einen weißen Ferrari. Die Farbe Weiß spielt in seiner Luxuswelt die entscheidende Rolle: sie steht hier für Reinheit und grenzt von den sonstigen Gangsta-Repräsentationen ab. Sie steht symbolisch für einen, der es geschafft hat und der sich für die nicht privilegierten Kinder einsetzt. Die Kamera behält die respektvolle Untersicht bei und umkreist den Sportwagen. Sie fährt anschließend auf Höhe des Seitenfensters mit, um die Worte des Rappers zu erfassen, der jetzt mit seinen tätowierten Armen aus dem Autofenster heraus seinen Rap gestisch begleitet. Die Fahrt in der Limousine verbindet die grüne Luxuswelt mit der normalen Neighbourhood, die repräsentiert wird durch das eingezäunte Basketballfeld, auf dem Busta Rhymes mit Anarchy-T-Shirt und weißem Bandanastuch um den 
Kopf inmitten von spielenden Kindern auftaucht. Busta Rhymes' Outfit ist in dieser Szene ein anderes, er zeigt sich solidarisch mit der Neighbourhood. Die Kamera geht auf Augenhöhe der Kinder, Rhymes duckt sich, nimmt die Perspektive der Kinder ein und erzählt von Prostitution und Glücksspiel in der Hood und ermutigt die Kinder dagegen vorzugehen. Die Zeichen des Gangstatums sind hier sehr dezent und neutral dargestellt: an der Ecke stehende, würfelnde Männer und eislutschende (einen »blowjob« andeutende) »chicks«, die für Prostitution stehen. Die Kinder laufen mit Rhymes und werden so zu seiner »posse «. Die Kamera übernimmt die Position der Kinder, die sich die Mittel der Erwachsenen aneignen: einen ÜberwachungsLKW mit Inhalt. Die Szene wird durch ein Close-up eingeleitet. Die Tür wird geöffnet, das Zoom holt Gegenstände im Inneren wie z.B. Handschellen näher heran. Die Kinder kostümieren sich mit Schnäuzern, Sonnenbrillen und Schlagstöcken. Der bewegte Schlagstock eines >Polizisten-Kindes erscheint in der Nahaufnahme und vertreibt die Glücksspieler. Im Parallelschnitt zu Busta Rhymes entspricht dem Schlagstock der deutende Zeigefinger. Die Kinder tragen dann Anzüge und übernehmen als verkleidete Erwachsene mit FBI-Ausweisen die Säuberung des Viertels. Sie werfen alle Prostituierten, Drogendealer und Zuhälter raus, indem sie eine Razzia an der Straßenecke und in einem Club veranstalten, wo sie die Gangster am Kragen hinauszerren. Vor dem Clubeingang steht ein Polizeiwagen am rechten Bildrand, der Zoom erfasst gestikulierende Polizisten. Der aus dem Bild fahrende Wagen des NYPD, der New Yorker Polizei, deutet an, dass die Polizei korrupt ist und nichts ausgerichtet hat. Zugleich ist dieses Auto auch der einzige Hinweis auf den Ort des Geschehens. Die zu bekämpfenden Zeichen nicht erwünschten Reichtums in der Neighbourhood zeigen sich im Club an Gegenständen wie Handys, Goldketten, teuer angezogenen Frauen, Bandanas und nach hinten gedrehten Baseballcaps. Diese Gegenstände, in anderen Videos positiv besetzt, werden zu negativen Zeichen und führen zum Verweis aus der Szenerie durch die Kinder. Die ganze Hood steht hinter dieser Aktion, Zwischenszenen zeigen die gesamte Nachbarschaft als Hintergrund für Busta Rhymes.

Das Video ist durch narrative Elemente bestimmt, nur wenige Stellen haben einen experimentellen Charakter: In Begleitung eines orange gekleideten Tänzers taucht Busta Rhymes zwischen dem blau-violett beleuchteten Club der Gangster und dem eigenen stilvollen, hellblauen Tanzclub in einem Gang auf, der mit Schwarzlicht beleuchtet ist, das inn bis zur Unkenntlichkeit blau einfärbt. Das Schwarzlicht trennt die Gangsterwelt von der korrekten Luxuswelt, die Platz für Kinder bietet und diese befähigt mit den Gefahren ihrer Umgebung aufzuräumen. 
Busta Rhymes ist als verbindende Hauptfigur zwischen den Teilen fast ausschließlich frontal vor der Kamera zu sehen. Er bewegt sich ständig auf die Kamera zu, entweder gehend oder mit den Gesten der Arme, die einen Raum erschließen. Die vertikalen Bewegungen im Bildraum sind für die Halbwelt und ihren Verweis aus dem Bild reserviert. Die Bilder des vorgestellten Videos sind sehr traditionell. Die Handlung läuft beinahe chronologisch ab. Auch die Schnitte der Parallelhandlungen strukturieren ein wenig, unterbrechen das lineare Band jedoch nicht. Das Tempo ist sehr niedrig. Es handelt sich hier eher um einen Kurzfilm, ein Eindruck, den die Beschränkung des Formats mit weißen Balken auf 16:9 zusätzlich unterstützt.

\section{Aphex Twin: »Windowlicker« (1999)}

Ganz anders und aus der Perspektive des weißen Zuschauers und Produzenten präsentiert sich das Video zu »Windowlicker «, einem Track mit elektronischer Musik ohne Text, in dem der Regisseur Chris Cunningham mit Masken des Gesichts von Aphex Twin arbeitet. Der Plot stellt eine Parodie des Gangsta-Genres im HipHop dar: Zwei schwarze Männer fahren mit ihrem Cabrio durch das sonnenreflektierende Los Angeles und reden über Sex. An einer Straßenecke stehen zwei schwarze Frauen in einem sexy Outfit, weißen Hot Pants und Bustiers. Die >Hormon-Gesteuerten< drehen ihr Auto und halten neben den Frauen an, um sie aufs Unverschämteste anzumachen. Die Frauen zeigen einen im HipHop-Genre selten vorkommenden oppositionellen Frauentyp, wie man ihn von Eve oder Foxy Brown kennt, der trotz knapper Bekleidung unmissverständlich klarmacht, dass man sich dumme Anmache nicht gefallen lässt. Die beiden Frauen schlagen verbal zurück und fragen, was die beiden »poor ass niggers « denn zu bieten hätten - weder Geld noch Stil: »look at your hair«. Sie lachen die beiden Hormon gesteuerten Männer aus. Im nächsten Moment wird das Cabrio von einer langen weißen Stretchlimousine weggeschoben, die sekundenlang unterhalb der eingefrorenen Gesichter der Frauen die Mitte des Bildes durchrollt und die beiden Männer im Nebel zurücklässt. Die staunenden Gesichter der Frauen, die sich herunterbeugen, spiegeln sich in dem Moment in der Scheibe, als diese von dem weißgekleideten Aphex Twin im Inneren der Limousine heruntergelassen wird. Er steigt aus und startet in einer Pfütze einen abstrusen Tanz, eine Mischung aus surrealem Ballett und einer Parodie der Michael JacksonSchritte aus dem Video »Beat It«. Dabei setzt er einen Schirm ein, der mit dem Aphex Twin-Logo bedruckt ist, das sich auch auf der Limousine befindet. Während die Frauen die Tanzeinlage bewundern, verwandeln sich ihre 
Gesichter in das vollbärtige Gesicht von Aphex Twin. Die nächste Szene zeigt das wilde Treiben der >Aphex-Drillinge<, die übereinanderliegenden üppigen Körper der Frauen, die im Inneren der Limousine Champagner trinken. Die vollbärtigen Frauen mit übertriebenen Afrolook-Frisuren umschmeicheln den Gastgeber und umkreisen ihn mit ihren Oberweiten.

Die von ihren Trieben beherrschten Männer im Cabrio bemerken die Verwandlung aufgrund ihrer Fixierung auf die weiblichen Körper nicht und folgen der Limousine. Die beiden Transgender-Klone mit dem männlichen Kopf und dem weiblichen Körper schauen aus dem Schiebedach und winken. Als nächster Ort wird die Strandpromenade in Szene gesetzt.

Dort formiert sich eine surreale Tanzgruppe mit Aphex Twin als VorTänzer. Die vollbusigen Transgender-Wesen vervielfältigen sich hier zu einem multikulturellen Tanzensemble. Die aufreizend tanzenden Bikinischönheiten stoßen den Vortänzer weg, um die beiden Cabriofahrer mit einer Blumenkette zu bekränzen. Aphex Twins magerer Körper wird im Tanz kontrastriert durch die üppigen Formen der Gogotänzerinnen unterschiedlicher Hautfarben, die in Anspielung auf akrobatische HipHop-Gruppentänze unpassende Bewegungen vollführen. Die nächste deutliche Anspielung auf die Geschichte des populären Tanzes ist die Szene, in der die Klone mit dem Aphex Twin-Schirm posieren. Hier wechselt die Kameraperspektive, um die Choreographie der Klone von oben zeigen zu können. Die nächste Tanzfigur, die die vollbusigen Vollbärtigen vollführen, erinnert an Busby Berkeleys Musicals. Neben den Schirmen werden jetzt auch - wiederum von oben gefilmt - Cheerleader Pompons geschwungen. Dann verlässt die Kamera die Vogelperspektive und nach einer Drehung verwandelt sich das Aphex Twin-Gesicht einer der Transgender-Schönheiten in ein groteskes weibliches Clownsgesicht mit Zahnlücken und Zöpfen. Die Cabriofahrer schrecken zurück und flüchten.

Alle visuellen Anspielungen auf das »Playa Prinzip« (Kodwo Eshun) in den HipHop-Gangsta- und R'n'B-Videos, Inbegriff für die sexistischen Bilder mit wenig bekleideten verobjektivierten Frauen und Luxus, weisen Cunningham als exzellenten Kenner der hier üblichen Stereotypen aus. Er treibt diese Klischees auf die Spitze. Kein sexistisches Element aus den G-Funk-Videos wird ausgelassen. So posiert der Twin mit einer geschüttelten Champagnerflasche, deren Inhalt sich auf den wogenden Körpern und Hinterteilen entlädt und alle benetzt. Dutzende von HipHop-Videos betonen immer wieder die männliche Potenz und den erfolgreichen männlichen Hyperorgasmus anhand dieser platten Symbolik (besonders extrem: LL Cool Js Video »Phenomenon«, 1998). 
Das Video zeigt Gender- und Race-Switching und unheimliche Transgender-Wesen, die bis zur clownesken Verzerrung mutieren und damit die geifernden triebfixierten Männer in Schrecken versetzen. Aphex Twin scheint der weiße Gewinner, der durch Stil, Luxus und Tanzkünste die Frauen beeindruckt und die schwarzen Poser abschreckt durch eine noch viel größere ästhetisch motivierte Pose. Aber nur oberflächlich gesehen ist es wieder ein Sieg des weißen Mannes über den schwarzen, wie Kaja Silvermann in der ZDF-Reihe Fantastic Voyages von 2001 konstatiert. Die Bilder geben eine andere Interpretation vor: Übertreibung und Verzerrung sind eine Parteinahme für die Transgender-Wesen, die die simpel strukturierten heterosexuellen Männer erfolgreich in die Flucht schlagen. Auch sind die hier gezeigten Künste weißer Beeindruckung pure Parodie: Der hagere Twin springt einen Laternenpfahl an und simuliert eine Kopulation. Dies ist eine abstruse Verdrehung des Tabledance, des Tanzens an der Stange. Auch das Durchziehen des geschlossenen Schirmes zwischen den Beinen ist eine Ableitung von weiblichen professionellen Anmachstrategien beim Striptease. Hinzu kommen groteske Beindruckungsposen und als Andeutung von großer Potenz wird der Schirm wild zwischen den Beinen hin und her geschwenkt.

Die Aphex Twin-Parodie zeigt eine groteske Verzerrung in der visuellen Verfügbarkeit des weiblichen Körpers als Objekt des Schauens. Die Enttäuschung des voyeuristischen Blicks wird stellvertretend durch die zwei schwarzen Männer vorgeführt und hallt im Zuschauer nach. In der gezeigten Konstellation von Körper und Gesicht wird die sonst unterschwellige pornographische Abtrennung nun in den Mittelpunkt des Bildes gestellt. Normalerweise ist das weibliche Gesicht in den G-Funk-Videos unwichtig, die Betonung liegt auf den Körperteilen, der sezierende Blick der Kamera präsentiert die verfügbaren Waren-Angebote (Mercer 1999: 440) des weiblichen Körpers. Durch die Metamorphose des Gesichts in »Windowlicker « wird die Abtrennung bewusst und damit rückgängig gemacht.

Dieses Video stellt eine Außensicht auf die HipHop-Klischees dar und konzentriert sich auf die Ästhetik. Der Clip beginnt wie ein Film mit der Kommunikation der schwarzen Männer und Frauen, das eigentliche Musikstück beginnt erst mit Erscheinen der Limousine. Es ersetzt die Kommunikation und lenkt die Konzentration auf die Bilder; es setzt sich mit den medial erzeugten Selbstbildnissen einer kommerziellen schwarzen Kultur auseinander und dekonstruiert die hier erzeugten Stereotypen ironisch.

Die Analyse des nächsten Clips führt zurück in eine schwarze Mainstream-Kultur. 


\section{Brandy: »What About Us« (2002)}

Der Text des R'n'B-Stücks ist simpel, er beschreibt die Unzufriedenheit der Sängerin mit einer Beziehung. Sie beschwert sich über die vielen Versprechungen, die nicht eingelöst werden und zeigt, dass viele andere gut aussehende Männer an ihr interessiert sind. Die Bilder sind im Verhältnis dazu spektakulär, es handelt sich um ein mit aufwendigen digitalen Effekten produziertes Video mit künstlichen Fantasy-Elementen.

Der Clip beginnt mit dem Close-up eines Zettels in altertümlicher Schrift: "Promise - to love you forever, - never to cheat, - always be there . Nach einem Schnitt ist Brandy auf der rechten Seite zu sehen, den Hintergrund bilden Pyramiden in einer grünen Landschaft. Die computergenerierte Landschaft ist unbestimmt, ortlos. Brandy hält zunächst die Sätze mit den gebrochenen Versprechen als Schrifttafel/-rolle in der Hand, dann hockt sie vor einer Schatztruhe. Diese befindet sich oben auf der Pyramide in einer Art Nest. Im Hintergrund werden fliegende Bäume mit breitem Wurzelwerk sichtbar, die eine Insel bilden. Auf einigen dieser Inseln schweben einzelne Männer auf Brandy zu und schauen sie an. Brandy trägt ein schwarzes Lederkostüm, schwarze lange Handschuhe und einen Nietengürtel. Sie greift in die rosenbekränzte Schatztruhe und holt ein Amulett hervor. Man sieht anscheinend die Schätze aus ihrer Kindheit, symbolisiert durch ein Stofftier, einen Affen. Die Schatztruhe steht für die Gegenstände aus der Vergangenheit, die positive Erinnerung. Sie schließt die Truhe, nun werden die Pyramiden wieder sichtbar und es stellt sich heraus, dass diese aus Männerkörpern bestehen. Die Körper schwarzer Männer sind symmetrisch angeordnet und mit Asche bemalt, einzelne Männer verziehen leidend das Gesicht. Später wird deutlich, dass sich diese Landschaft in einer fernen Galaxie befindet.

Nach einem für die digitalen Gestaltungsmittel typischen Flug über die grüne Landschaft und einen See folgt der Betrachter der Kamera in einen sTimetunnel<, der aus technoiden Elementen und schwarz-silbergrauen Lamellen zusammengesetzt ist. Hier steht Brandy, sie trägt ein Leder-NietenKostüm und Stiefel mit Fransen, tanzt durch den Tunnel und schwenkt ihre Hüften, macht Bewegungen wie beim Kickboxen. Dann fliegt ein Baseballschläger heran. Brandy fängt ihn zielsicher auf, betrachtet inn mit sichtlichem Vergnügen und bezieht ihn in den Tanz mit ein. Sie schwingt den Baseballschläger rhythmisch hin und her und steht, bereit für einen gezielten Schlag, breitbeinig auf ihren hochhackigen Stiefeln. In Großaufnahme wird Brandys ebenmäßiges Gesicht gezeigt, das sie wie eine computergenerierte 
digitale Schönheit erscheinen lässt. Die Kamera fährt zurück: Ein Handy fliegt auf Brandy zu, sie befeuchtet einen Finger mit der Zunge, hält inn hoch als Geste des Zielens und schlägt das Handy zurück in den Tunnel. Die nächste Grossaufnahme zeigt einen fliegenden PDA mit einer schemenhaft erkennbaren »message for Brandy« auf dem Display. Auch dieser Gegenstand wird gekonnt zurückgeschlagen. Der Spaß an der Aktion wird daran verdeutlicht, dass der Schlag wiederholt wird, nun zeigt die Kamera ihn von der anderen Seite. Der PDA fliegt in zwei Teile zerschlagen zurück, Brandy tänzelt mit Baseballschläger und gibt sich sportlich. Als Nächstes fliegt kopfüber ein Mann in hellblauem Nicki-Jogginganzug vorbei: der Verflossene; hier kommt der Baseballschläger nicht zum Einsatz. Brandy nimmt ihm im Vorbeiflug die Sonnenbrille ab und setzt sie auf. Es zeigt sich eine Spiegelung in den Gläsern der Sonnenbrille, die den morphigen Übergang in einen neuen Raum bildet. Es ist ein silberner, digital erzeugter Raum mit einer Gitterplattform in Form einer trichterförmigen Wäschespinne in der Mitte, in der sich die Sängerin, gekleidet im roten Kleid mit bodenlangen Fransen und schwarzen Handschuhen, befindet. Um sie herum winden sich zwei glänzende Graphitmänner in Ketten, die in Close-ups ihre Waschbrettbäuche, die muskulösen Körper und ihre glänzende Haut zur Schau stellen. Die Männer tragen Nietenbänder um den Hals, sie knien, beugen den Kopf herunter und strecken ihre Hände nach vorne. Die schwarzen Tänzer bewegen sich vor einem Hintergrund aus bewegten Silberstreifen. Der Boden des Gittertrichters ist mit in Falten gelegtem schwarzen Satin ausgelegt.

Der nächste harte Schnitt führt zurück in das Land mit den schwebenden Bäumen und den Männerpyramiden, diesmal im Lichte eines >Sonnenuntergangs . Brandy trägt wieder die schwarze hautenge Lederkombination mit Hüfthose. Sie blickt nach oben und es wird deutlich, dass sie sich auf einem anderen Planeten befindet. Sie sieht die Erde und den Mond über sich. Die Kamera fährt nach oben und zoomt auf die Erdkugel; die Blickrichtung dreht sich und aus der Vogelperspektive werden gleichmäßige bunte, rechteckige Strukturen sichtbar. Je weiter das Zoom Richtung Erde geht, desto deutlicher wird, dass es sich um einen riesigen Parkplatz mit Autos handelt. Die Kamerafahrt endet mit einem Close-up, das Brandy in einem Cabrio, einem alten Straßenkreuzer aus den 1950er Jahren mit verchromten Stoßstangen, zeigt. Ein Mann in rotem Muskelshirt schaut zu ihr herüber, weitere Autos mit männlichen Insassen werden gezeigt, ihre Gesichter sind angeschnitten, sie lümmeln sich cool in ihren Autos. Es sind die Männer von den schwebenden Bäumen auf dem Planeten. Dann wirft die Kamera einen Blick von oben auf Brandys Auto. Sie hält eine Jumpcar-Fernsteuerung in der Hand, mit der sie das Auto langsam zum Tanzen bringt. Über ihr wölbt sich der schwarze 
Himmel mit Vollmond. Brandy sswingt < im Auto hin und her und steuert die Hydraulik; das Auto ruckelt. Schließlich steigt ein Typ in schwarzem LederOutfit zu Brandy, die Sängerin grüßt ihn mit einer lässigen Bewegung des Kopfes und einem kühlen, selbstbewussten Lächeln.

Brandy ist zeitgemäß gekleidet, ihre Mode erweist der ausklingenden Cowboymode und dem Punk-Revival Referenz. Die Männer auf den schwebenden Inseln und die Typen in den Autos tragen gängige HipHop-Outfits: z.B. Kopfbedeckungen wie Bandanas, Beanies und Anglerhüte.

Das Video zeigt verschiedene Orte und Zeitebenen. Die digitalen Effekte, wie das Metallische im Silberraum, die Graphitkörper der Tänzer, Brandys Lippen und die Tunnelfahrten erzeugen die sfluide< Ästhetik des Videos, die durch harte Cuts kontrastiert wird. Immer wieder wechselt die Perspektive von Close-ups der Sängerin bis zu Großaufnahmen ihres Munds mit grell geschminkten roten Lippen oder der schwarz geschminkten Augen. Ihr Gesicht und ihr Körper sind hierbei künstlich wie die Landschaft und verstärken den extrem artifiziellen Eindruck des gesamten Videos.

Die Bilder zeigen die Bestimmtheit einer minimalen autonomen weiblichen Haltung, die Auswahl des Partners selbst zu treffen und sich nicht einwickeln zu lassen. Der Baseballschläger weist auf diese Bestimmtheit hin und nicht etwa auf ihre Absicht, die Männer für ein an ihr begangenes Unrecht körperlich zu züchtigen, wie es z.B. in Kelis' Video zu »Caught You Out There « geschieht.

Brandys Video repräsentiert eine Arbeit an den Geschlechterverhältnissen im Kleinen, sie emanzipiert sich im Beziehungsalltag von leeren männlichen Versprechungen von Ewigkeit, Anwesenheit und Treue, Themen, die in den Texten der HipHop-Ladies immer wieder auftauchen. Sie bewegt die Jumpcar-Hydraulik selbst, dies ist sonst in G-Funk-Videos Teil der männlichen Verführungsstrategie, um Potenz und Steuerungsgewalt zu demonstrieren. Sie eignet sich diese technischen Accessoires an und gleichzeitig zeigt sie die Ablehnung von (Kommunikations-)Technologien als Mittel, falsche Versprechungen zu transportieren. Im Kontrast dazu sind ursprüngliche, noch nicht gebrochene Versprechungen klassisch auf Schrifttafeln festgehalten.

Planet und Silberraum zeigen die Utopie einer weiblichen Herrschaft über die Männer bis zur Versklavung, die Männer stehen zur Verfügung und sind formbares Material. Diese Orte geben Hinweise auf eine mögliche matriarchalische Zukunft, in der die Frauen als Königinnen auf den Männerbergen residieren und in der Versprechungen eingehalten werden müssen. Auch zeigt sich hier das Prinzip der besitzergreifenden bergeweisen Akkumulation der Körper, die sonst den Männern vorbehalten ist. Der Versuch der Über- 
tragung auf die Erde, verbildlicht in der Umkehrung der Kamerafahrt auf die Erde, ist schwierig; lediglich die Jumpcar-Steuerung enthält den Hinweis auf eine autonome Zukunft.

Brandy präsentiert sich als sanfte Rebellin, die männlich konnotierte Alltagsgegenstände besetzt und für die Eroberung eines Mannes einsetzt, um sich dann wieder unterzuordnen. Der Jumpcar-Mechanismus wird von ihr nicht mehr benutzt, als der Mann im Auto sitzt. Auffällig ist, dass nun das Verdeck des Cabrios geschlossen ist und es wird deutlich, dass die Sängerin die ganze Zeit auf dem Beifahrersitz gesessen hat. Die Demonstration von totaler Autonomie wird in diesem Moment ausgesetzt und zur zukunftsfernen Utopie.

\section{Brandy, Busta und das dritte Geschlecht}

Bei Brandy wie auch bei Busta Rhymes handelt es sich um ein MainstreamR'n'B-Videos. Sie sind daher gut vergleichbar. Busta Rhymes' Schwerpunkt ist die Solidarität mit der Neighbourhood, Frauen und ihre Sexyness stehen nicht im Vordergrund, hier werden nur die negativen Beispiele, die »chicks« gezeigt. Ein Kind, keine Frau, bewohnt mit ihm das Schlosshaus, er zeigt sich als alleinerziehender Vater, eine seltene Rolle im HipHop-Video. Er verteidigt die Rechte der Kinder gegen die Gangsta. Es geht um die Darstellung von männlicher Selbstbestimmung ohne Macho-Attitude.

Beide Videos sind auf den ersten Blick nicht spektakulär. Sie zeigen keine slauten Bilder, sind aber trotzdem entweder sehr aufwendig digital gestaltet, oder präsentieren, wie im zweiten Fall, eine relativ dichte Erzählung. Brandy stellt sich nicht als eine aggressive wilde Frau dar, die ihre sexuellen Phantasien beschreibt und im Video auslebt wie etwa Lil' Kim. Sie ist sexy gekleidet, ohne sich total zu entblößen. Brandy ist kein HipHopSupervamp, keine »bitch«, ihre Kleidung ist weder aufreizend, noch androgyn oder komödiantisch wie die von Missy Elliott. Sie steht für ein sauberes Beziehungsangebot.

Die drei analysierten Videos versinnbildlichen unterschiedliche Räume: Busta Rhymes konzentriert sich auf den Nahraum, die Neighbourhood. Ein lokaler Bezug taucht auch peripher bei Aphex Twin auf, sein urbaner Raum wird durch überzogene männliche Gestalten besiedelt und zeigt die selbstbewussten schwarzen Ladies. Ein zweiter Raum wird nach dem »Playa Prinzip« in der Luxuslimousine und auf dem Strandplateau eröffnet. Das Video hat ein durchgehendes Design und präsentiert die Marke >Aphex Twin <, die alle sexuellen Versprechungen nicht einlöst und den weißen Voyeur sowie 
die männlichen schwarzen Protagonisten enttäuscht. Brandy präsentiert sich in virtuellen Wunsch(t)räumen. Planet und Silberraum stehen für die weibliche Zukunft, die Schatztruhe für eine erfüllte Vergangenheit. Ihr Video ist klinisch und repräsentiert eine künstliche Erotik. Die visuelle Verknüpfung der >fluiden< Räume mit der Figur des »verrückten Niggas« wird bei Brandy aufgebrochen.

Alle drei Videos zeigen eine Mischung aus Story und Performance der Künstlerin bzw. des Künstlers. Sie folgen einer narrativen Struktur und ihre Bilder sind eng mit den Textaussagen verknüpft. Die Videos orientieren sich an Motiven und Erzählungen der HipHop-Kultur, gleichzeitig stellen sie Differenz zu den üblichen Bildern her. Eine zentrale Rolle spielen auch in den ausgewählten Beispielen Referenzkategorien wie Luxus und Reichtum. Diese zeigen sich u.a. konkret an der Kleidung, die auf die Ursprungsszene verweist, aber darüber hinausgeht. Alle Videos bedienen sich der aussagekräftigen und gleichzeitig stereotypen Farben Schwarz und Weiß im Outfit der Darsteller: Busta Rhymes trägt weiße Sachen zu Beginn des Videos, Aphex Twin selbst und seine Klone, die schwarzen Frauenkörper, tragen ebenfalls weiß. Busta Rhymes und Aphex Twins Limousinen sind weiß. Bei »Windowlicker« wird vor allem das klischeehafte Moment, dass der schwarze Körper am besten mit weißer Kleidung kontrastiert wird, durch den bleichen Aphex Twin konterkariert. Brandy entscheidet sich dagegen für Schwarz, um ihre besondere weibliche Rolle hervorzukehren, die Konnotation Luxus ist bei ihr nicht zentral.

Weiterhin ist vor allem die herausragende Bedeutung des Autos als Verweis auf Mobilität zu nennen. Besonders deutlich werden die unterschiedlichen Perspektiven der drei Beispiele an einem Detail: der Präsentation des Autoraumes. Der Autoinnenraum bei Brandy wandelt sich von dem offen autonomen des Cabrios in einen geschlossenen, in dem der Mann wieder am Steuer sitzt. Die Blick-Undurchlässigkeit der Stretch-Limo(usine) in Form der verspiegelten Scheiben wird durch das offene Schiebedach konterkariert, aus dem die >Aphex-Drillinge < winken. Die heruntergekurbelten Scheiben des Luxusautos von Busta Rhymes deuten ebenfalls auf eine offene Haltung und ein Absehen von einer PS-verstärkten Machohaltung. Der Macht- und Verführungsort im HipHop-Video erfährt hier Variationen, die Auskunft über die zugrundeliegende Geschlechterbilder geben und neue Konstellationen entwerfen: der Mann, der Verantwortung für die Kinder der Hood übernimmt, die schwarzen Machos, die sich von Misch-Geschlechtern verwirren lassen, und eine Emanzipation der kleinen Schritte, die die weibliche Zukunft auf einen anderen Planeten verlegt. 


\title{
Literatur
}

Crouch, Stanley (1993). »Man in the Mirror.«In: Diederichsen 1993: 149-154.

Decker, Jeffrey Louis (1994). »The State of Rap: Time and Place in HipHop Nationalism. «In: Rose/Ross 1994: 99-121.

Diederichsen, Diedrich (Hg.) (1993). Yo! Hermeneutics! Schwarze Kulturkritik. Pop, Medien, Feminismus. Berlin: Edition ID-Archiv.

Mercer, Kobena (1999). »Reading Racial Fetishism: The Photographs of Robert Maplethorpe.« In: Visual Culture: The Reader. Hg. v. Stuart Hall und Jessica Evans. London: Sage, S. 435-447.

Hooks, Bell (1992). Black Looks. Race and Representation. Boston: South End Press. Hooks, Bell (1993). »Schwarze intellektuelle Frauen.«In: Diederichsen 1993: 39-49.

Julien, Isaac (1993). »Black Is, Black Ain't. Bemerkungen zur De-Essentialisierung schwarzer Identitäten. «In: Diederichsen 1993: 217-224.

McLaren, Peter (1995). Gangsta Pedagogy and Ghettocentricity: The HipHop Nation as Counterpublic Sphere. Los Angeles: als Manuskript.

Richard, Birgit (Hg.) (1998). Die Hüllen des Selbst. Mode als ästhetisch-medialer Komplex (= Kunstforum International Bd. 141, Juni-September). Ruppichteroth: Kunstforum-Bücherdienst.

Rose, Tricia / Andrew Ross (Hg.) (1994): Microphone Fiends: Youth Music and Youth Culture. New York, London: Routledge.

Rose, Tricia (1994a). »A Style Nobody Can Deal With.«In: Rose/Ross 1994: 71-81.

Rose, Tricia (1994b). »Contracting Rap: An Interview with Carmen AshhurstWatson. « In: Rose/Ross 1994: 122-144.

Shusterman, Richard (1994). Kunst Leben. Die Ästhetik des Pragmatismus. Frankfurt/M.: Fischer.

Tate, Greg (1993). »Die Liebe und der Feind. « In: Diederichsen 1993: 225-228.

Wallace, Michele (1993). »Black Macho - Damals und Heute.« In: Diederichsen 1993: 55-69.

\begin{abstract}
This contribution concentrates on the pictorial construction of the identity of the black subject and its spacial context. The special focus is on the representation of gender in search for emancipatory images of the female in contemporary HipHop clips. Even images and surfaces that appear sexistic at first sight may be re-occupied and transformed into an autonomous female imagery. The selected clips show the ambivalent construction of gender most clearly. They exemplify a critical view on the stereotype of gangsterism, a form of parody constructed from a white point of view and the in-between spaces for a female gaze.
\end{abstract}

